\title{
$i$-Steps Closed-Loop Sets for Constrained Linear Systems under Model Predictive Control
}

\author{
Alejandro Anderson \\ INTEC-CONICET \\ Universidad Nacional del Litoral \\ Santa Fe, Argentina \\ alelanderson@gmail.com \\ Ernesto Kofman \\ Deparatmento de Control \\ FCEIA UNR and CIFASIS-CONICET \\ Rosario, Argentina \\ kofman@fceia.unr.edu.ar
}

\author{
Agustina D'Jorge \\ INTEC-CONICET \\ Universidad Nacional del Litoral \\ Santa Fe, Argentina \\ agustinadj@gmail.com
}

\author{
Antonio Ferramosca \\ CONICET - UTN \\ Facultad Regional de Reconquista. \\ Reconquista, Argentina \\ ferramosca@santafe-conicet.gov.ar
}

\begin{abstract}
The understanding of invariant set theory is essential in the design of controllers for constrained systems. This paper presents some concepts related with the invariant set theory and Set-Based Model Predictive Control (set-based MPC). Precisely, introduces a new class of sets from where the closedloop system reaches a target set before a pre-established number of steps. These novel concepts are based on several results presented in a former work [1].

The main results are exposed in a theoretical context, however several simulation examples show its potential and properties.

Index Terms-Model Predictive Control, Finite Time Convergence, Controllable Sets, $i$-steps closed-loop sets.
\end{abstract}

\section{INTRODUCTION}

Model Predictive Control (MPC) is probably the most employed advanced control technique in process industries. One of the reason for such success is its ability to incorporate, from the beginning, constraints on both, control inputs and states/outputs [2]. From a theoretical point of view, the invariant set theory has been successful in providing sufficient conditions for nominal and robust feasibility/stability [3], [4], permitting the analysis of stabilizable regions and generalizing the stability of equilibrium points to stability of invariant sets. Furthermore, this theory turned out to be very useful for the analysis of dynamical systems subject to constraints, since the concept of positive invariance is closely related to Lyapunov theory [4], [5].

Although the results on stability of MPC are mainly devoted to prove asymptotic (or exponential) stability, there are a few results on finite-time convergence for a fixed control horizon [1], [6], [7]. In particular, [1] proposes a set-based MPC in which the target/objective is given by an arbitrary small invariant set and its corresponding input set - that ensures finite-time convergence and also provides an upper bound for the time of convergence.

Based on the upper bound provided by [1], the state space can be partitioned into nested regions, which are called the $i$ steps closed-loop set; i.e., a particular regions from where the closed-loop system reaches the target set in $i$ or less steps. The similarity with the concept of the (open-loop) $i$-steps controllable sets from the classic literature ( [5], [8]) is evident. The $i$-steps controllable sets indicates the region from where the open-loop system could reach the target set in a given number of steps, by means of feasible control actions and by a feasible trajectory. The novel concept of $i$-steps closed-loop set is introduced with the hope that the formal framework presented in this note will facilitate a better understanding of the most basic problem in the design of MPC controllers.

The paper is organized as follows. Section II states the problem and presents some preliminary definitions. The proposed MPC scheme, the finite time convergence results and a proper upper bound for this convergence are presented in Section III. Section IV introduces the main results of the work. Section V shows some simulations. Finally, the conclusions are given in Section VI.

\section{A. Notation}

The integers set is defined by $\mathbb{I}$ and the integers between $N$ and $M$ by $\mathbb{I}_{N: M}:=\{N, N+1, \ldots, M\}$. Given any real number $x \in \mathbb{R}$, the floor of $x$ is defined by $\lfloor x\rfloor:=\max \{n \in$ $\mathbb{I}: n \leq x\}$. Let $\gamma \in \mathbb{R}$ be a scalar and a set $\Omega \subset \mathbb{R}^{n}$; the scaled set is defined as $\gamma \Omega:=\{\gamma x: x \in \Omega\}$. The boundary of a closed set $\Omega$ is denoted by $\partial \Omega$. The distance from $x \in \mathbb{R}^{n}$ to the set $\Omega$ is defined as $d_{\Omega}(x):=\inf \{d(x, y): y \in \Omega\}$.

\section{PROBlem STATEMENT AND PRELIMINARY DEFINITIONS}

Consider a discrete time system described by a linear timeinvariant model

$$
x(k+1)=A x(k)+B u(k), \quad x(0)=x_{0},
$$

where $x(k) \in \mathscr{X} \subset \mathbb{R}^{n}$ is the system state at the $k$-th sample time, $x_{0}$ is the initial state, and $u(k) \in \mathscr{U} \subset \mathbb{R}^{m}$ is the current control input. All along this work it is assumed 
that matrix $A \in \mathbb{R}^{n \times n}$ has all its eigenvalues strictly inside the unit circle $^{1}$, the pair $(A, B)$ is controllable, the set $\mathscr{X}$ is convex and compact, the set $\mathscr{U}$ is convex and compact and both contain the origin in their interior.

\section{A. Preliminary concepts}

$\zeta$

The next definitions and properties will be referred to system (1), and the corresponding state and input constraints.

Definition 1. (One-step controllable set) Given the set $\Omega \subset$ $\mathscr{X}$, the one step controllable set to $\Omega, \mathscr{Q}(\Omega)$, is the set of all $x \in \mathscr{X}$ for which there exists $u \in \mathscr{U}$ such that $A x+B u \in \Omega$, i.e.,

$\mathscr{Q}(\Omega):=\{x \in \mathscr{X}: \exists u \in \mathscr{U}$ such that $A x+B u \in \Omega\}$.

This is the set of states in $\mathscr{X}$ for which a control does exist in $\mathscr{U}$, such that the system can be steered to $\Omega$ in one time step. Furthermore, the concept can be generalized to the $i$-steps controllable set $\mathscr{Q}^{i}(\Omega)$, for any $i \in \mathbb{N}$, by applying the above definition iteratively, i.e., $\mathscr{Q}^{i}(\Omega):=\mathscr{Q}\left(\mathscr{Q}^{i-1}(\Omega)\right)$, for $i=1, \cdots, N$, and $\mathscr{Q}^{0}(\Omega):=\Omega$.

Definition 2. ( $\gamma$-control invariant set, $\gamma$-CIS) Given $\gamma \in(0,1], \Omega \subseteq \mathscr{X}$ is a $\gamma$-control invariant set if $x \in \Omega$ implies that $A x+B u \in \gamma \Omega$ for some $u \in \mathscr{U}$. Associated to $\Omega$, is the corresponding input set $\Pi(\Omega):=\{u \in \mathscr{U}: \exists x \in \Omega$ such that $A x+B u \in \gamma \Omega\}$.

Once the system enters to a $\gamma$-CIS, there is an admissible control input (belonging to $\Pi(\Omega)$ ) that is able to keep the system inside the set. When $\gamma=1, \Omega$ is simply a control invariant set.

It is known from [9] that every $\gamma$-CIS $\Omega$ is such that $\Omega \subseteq \mathscr{Q}(\Omega)$. In [1], the following stronger geometric property is presented:

Property 1. Let $\Omega \subset \mathscr{X}$ be a compact and convex $\gamma$-CIS, with $\gamma<1$ and the origin as an interior point, with the corresponding input set $\Pi(\Omega) \subseteq \mathscr{U}$. Then, $\Omega \subseteq \operatorname{int} \mathscr{Q}(\Omega)^{2}$.

\section{MPC SCHEME FOR FINITE-TIME CONVERGENCE}

In this Section, a general MPC formulation is presented. Given a fixed horizon $N \in \mathbb{N}$, and a compact and convex set $\Omega$, containing the origin in its interior, consider the following cost function:

$$
V_{N}(x, \Omega ; \mathbf{u}):=\sum_{j=0}^{N-1} L(x(j), u(j) ; \Omega)
$$

\footnotetext{
${ }^{1}$ If an original unstable matrix $A$ is given, then the system can be prestabilized by means of a stabilizing feedback law.

${ }^{2}$ Denote int $\mathscr{Q}(\Omega)$ the interior of the set $\mathscr{Q}(\Omega)$
}

where $L(\cdot) \geq 0$ is a general stage cost that depends in some sense on $\Omega$ (it could assume different forms according to each particular MPC formulation) and $\mathbf{u}:=\{u(0), \cdots, u(N-1)\}$. $\Omega$ can be thought as the objective or target set where the finitetime convergence has to be ensured to.

Let $\mathscr{U}_{N}(x)$ denote the set of admissible control sequences $\mathbf{u}=\{u(0), \cdots, u(N-1)\}$ satisfying the state and control constraints, together with a terminal constraints when the initial state is $x$; i.e., given an initial state $x=x(0)$, a sequence $\mathbf{u}=\{u(0), \cdots, u(N-1)\}$ with $u(j) \in \mathscr{U}$ such that $x(i) \in \mathscr{X}$, where $x(i+1)=A x(i)+B u(i)$ for $i=0, \cdots, N-1$, and $x(N) \in \Omega$. Specifically,

$\mathscr{U}_{N}(x)=$

$\left\{\mathbf{u} \in \mathscr{U}^{N}: x(j) \in \mathscr{X}, u(j) \in \mathscr{U}, j \in \mathbb{I}_{0: N-1}, x(N) \in \Omega\right\}$.

By Definition 1 , for all $i \geq 0, \mathscr{Q}^{i}(\Omega)$ denotes the set of states $x$ such that $\mathscr{U}_{i}(x) \neq \emptyset$, that can be steered to the target set $\Omega$ in $i$ steps by an admissible control sequence $\mathbf{u}$. At each time instant $k$, the MPC control law is derived from the solution of the following optimization problem:

$$
\mathscr{P}_{N}(x, \Omega): \min \left\{V_{N}(x, \Omega ; \mathbf{u}): \mathbf{u} \in \mathscr{U}_{N}(x)\right\},
$$

where $\Omega$ and the initial sate $x \in \mathscr{X}$ are the optimization parameters and the sequence $\mathbf{u}$ is the optimization variable.

The optimal state trajectory $\mathbf{x}^{0}(x)$, corresponding to the optimal control sequence $\mathbf{u}^{0}(x)$, is given by

$$
\mathbf{x}^{0}(x)=\left\{x^{0}(0 ; x), x^{0}(1 ; x), \ldots, x^{0}(N ; x)\right\} .
$$

By definition of $\mathscr{P}_{N}(x, \Omega), x^{0}(N ; x) \in \Omega$. The control law, derived from the application of a receding horizon policy, is given by $\kappa_{M P C}(x)=u^{0}(0 ; x)$, where $u^{0}(0 ; x)$ is the first element of the solution sequence $\mathbf{u}^{0}(x)$. This way, the closedloop system under the MPC control law is described as:

$$
x(k+1)=A x(k)+B \kappa_{M P C}(x(k)) .
$$

The Optimal Cost Function is given by the function $F(\cdot)$ which depends on the initial state, as follows:

$$
F(x):=V_{N}\left(x, \Omega ; \mathbf{u}^{0}(x)\right) .
$$

\section{A. Ingredients for finite-time convergence and upper bound}

Consider the following set-based stage cost:

$$
L(x, u ; \Omega)=d_{\Omega}(x)+d_{\Pi(\Omega)}(u)
$$

where the function $d_{\Omega}: \mathbb{R}^{n} \rightarrow \mathbb{R}$ is the distance from the state to the set $\Omega$, with a metric on $\mathbb{R}^{n}$ and $d_{\Pi(\Omega)}: \mathbb{R}^{m} \rightarrow \mathbb{R}$ is the distance from the control input to the set $\Pi(\Omega)$, with a metric on $\mathbb{R}^{m}$. The set $\Omega$ is assumed to fulfill the hypothesis of Property 1.

Remark 1. Note that according to this formulation, the control objective is considered to be reached once the system enters $\Omega$, and no further implicit objectives are considered. However, the interesting point is that the system will not be 
in open loop, since the controller will not allow the state to jump outside $\Omega$.

The following Lemma established in [1] presents the finitetime convergence and the corresponding upper bound for the time of convergence.

Lemma 1. (Upper bound) Consider the MPC formulation $\mathscr{P}_{N}(x, \Omega)$, (2), with the stage cost (5). Then, $\Omega$ is locally reached in finite-time by the system (3), with $x=x(0) \in$ $\mathscr{Q}^{N}(\Omega)$. Even more, the closed-loop system reaches $\Omega$ in at most $\left\lfloor K_{x}\right\rfloor$ steps, with ${ }^{3}$

$$
K_{x}=\frac{F(x)}{\delta}+1
$$

where $\delta=\min _{y \in \partial \mathscr{Q}(\Omega)} d_{\Omega}(y)$.

The importance of the upper bound $K_{x}$ lies on extending the idea of controllable set established in Definition 1 to closedloop systems, which is developed in the following section.

\section{IV. $i$-STEPS CLOSED-LOOP SETS}

There are many systems that must reach a safe region in a certain finite time. For instance, if it is desired for some variables to reach a basal value in less than, say, five steps, a classical MPC (or any other optimizing controller) cannot guarantee this requirement even if the system starts from $\mathscr{Q}^{5}(\Omega)$.

In this context, it is desirable to characterize another kind of sets that account for such closed-loop requirements. Let us define the following set, related to the proposed MPC closed-loop system:

Definition 3. (i-steps closed-loop set) The $i$-steps closed-loop set to $\Omega, \mathscr{C}^{i}(\Omega)$, is the set of all states $x \in \mathscr{X}$ such that $\left\lfloor K_{x}\right\rfloor \leq i$.

This definition is subject to the computation of the constant $K_{x}$ in equation (6) and its dependence on the initial state $x$. In order to get tighter sets the computation of $K_{x}$ must be less conservative.

\section{A. Characterization and properties of the i-steps closed-loop sets}

The following proposition gives a way to characterize the $i$-steps closed-loop sets.

Proposition 1. Let $i \in \mathbb{N}$. Consider $x \in \mathscr{X}$, fulfilling the constraint: $F(x)<\delta(i-1)$. Then, $x \in \mathscr{C}^{i}(\Omega)$.

\footnotetext{
${ }^{3}$ Note that $K_{x}$ is well defined since $\Omega \subseteq \operatorname{int} \mathscr{Q}(\Omega)$ by Property 1 , which means that $\delta>0$.
}

Proof. Suppose $x \in \mathscr{X}$ is such that $F(x)<\delta(i-1)$. Proving that $\left\lfloor K_{x}\right\rfloor \leq i$ is equivalent to show that $x \in \mathscr{C}^{i}(\Omega)$. Indeed,

$$
\begin{aligned}
\left\lfloor K_{x}\right\rfloor \leq K_{x} & =\frac{F(x)}{\delta}+1 \\
& \leq(i-1)+1 \\
& =i .
\end{aligned}
$$

Which concludes the proof.

Property 2. Let $\Omega$ be a CIS and $i \in \mathbb{N}$, then

(i) $\Omega \subseteq \mathscr{C}^{1}(\Omega)$

(ii) $\mathscr{C}^{i}(\Omega) \subseteq \mathscr{C}^{i+1}(\Omega)$ and

(iii) $\mathscr{C}^{i}(\Omega) \subseteq \mathscr{Q}^{i}(\Omega)$.

Proof. (i) If $x \in \Omega$, then $F(x)=0$, which means that $K_{x}=1$. (ii) If $x \in \mathscr{C}^{i}(\Omega),\left\lfloor K_{x}\right\rfloor \leq i \leq i+1$, then $x \in \mathscr{C}^{i+1}(\Omega)$. (iii) Let $x \in \mathscr{C}^{i}(\Omega)$. The optimal sequence of control drives the state $x$ to $\Omega$ in about $i$ steps (or less). So, the fact that $x$ can be steered to set $\Omega$ in $i$ steps means that $x \in \mathscr{Q}^{i}(\Omega)$.

Remark 2. In general, the i-steps controllable sets depend only on the system and the constraints, and they are independent of the controller properties. On the other hand, it is interesting to note that the i-steps closed-loop sets are based on the Optimal Cost Function, $F(x)$. Furthermore there is an intrinsic relation between controllable sets and $i$-steps closed-loop sets according to Property 2.

\section{Simulations}

In this section, the differences between the $i$-controllable sets $\mathscr{Q}^{i}(\Omega)$, which were extensively studied in the MPC literature, and the $i$-steps closed-loop sets $\mathscr{C}^{i}(\Omega)$ presented in this work, are demonstrated by a detailed simulating example.

\section{A. Simulation parameters}

The simulated system is similar to the one presented in [10], it is a second order stable linear system, given by:

$$
x(k+1)=A x(k)+B u(k),
$$

with

$$
\begin{aligned}
& A=\left[\begin{array}{cc}
0.7476 & -0.4984 \\
0.0356 & 1.0680
\end{array}\right], \\
& B=\left[\begin{array}{c}
0.3 \\
-0.4
\end{array}\right]
\end{aligned}
$$

The constraints of the system are given by $\mathscr{X}=$ $\left\{x \in \mathbb{R}^{2}:\|x\|_{\infty} \leq 10\right\}$ and $\mathscr{U}=\left\{u \in \mathbb{R}:\|u\|_{\infty} \leq 4\right\}$. The horizon of the MPC controller $\mathscr{P}(x, \Omega)$, is given by $N=7$, being $\Omega$ a $\gamma$-CIS with $\gamma=0.65$, and its corresponding input set $\Pi(\Omega)=\left\{u \in \mathbb{R}:\|u\|_{\infty} \leq 3\right\}$.

The distances involved in the stage cost (5) are define by $d_{\Omega}(x)=\inf \left\{(x-y)^{\prime} R(x-y): y \in \Omega\right\}$ and $d_{\Pi(\Omega)}(u)=$ $\inf \left\{(u-v)^{\prime} Q(u-v): v \in \Pi(\Omega)\right\}$, with $Q$ and $R$ being positive definite matrices. Different parameters of $Q$ and $R$ are 
used to perform the simulations in order to test the variability on the $i$-steps closed-loop sets according the changes on the optimal cost function. Simulations on Figure 1 and 4 are performed with $R$ and $Q$ as identity matrices and Figure 2 and 5 with $R$ as an identity matrix multiplied by 100 , and $Q$ as an identity matrix.

\section{B. $i$-Steps controllable sets}

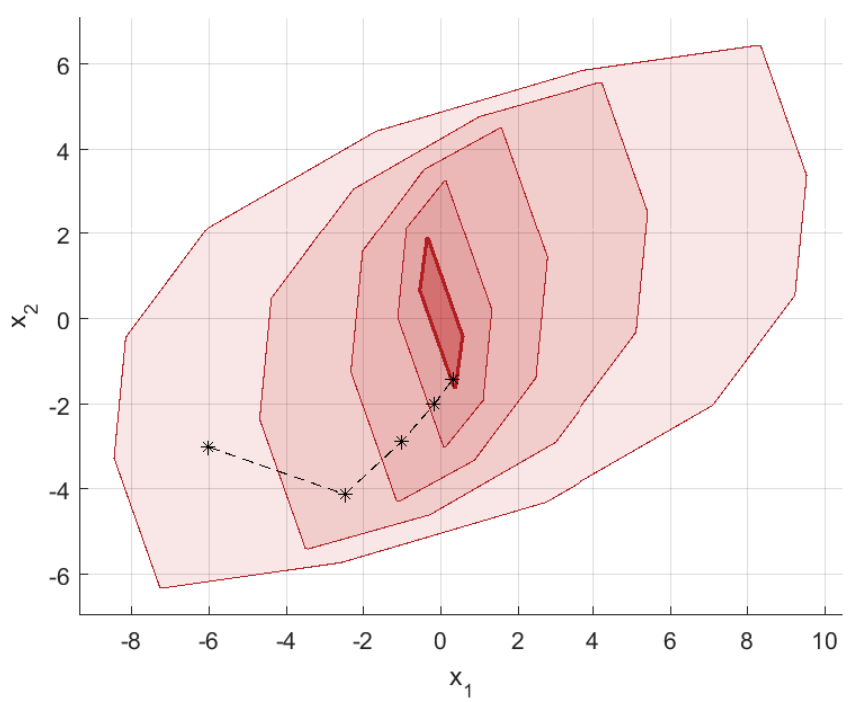

Fig. 1: The $\gamma$-CIS $\Omega$ and its controllable sets $\mathscr{Q}^{i}(\Omega)$ for $i=1, \ldots, 4$. The controlled system with $x_{0}=(-6,-3) \in$ $\mathscr{Q}^{4}(\Omega)$.

Figure 1 shows the $\gamma$-CIS $\Omega$ and its corresponding $\mathscr{Q}^{i}(\Omega)$ for $i=1,2,3,4$. Besides, the initial state $x_{0}=(-6,-3)$ belongs to $\mathscr{Q}^{4}(\Omega)$ - is controlled by the MPC controller derived from $\mathscr{P}(x, \Omega)$, which means (by Lemma 1 ) that the target set is reached in a finite number of steps. In this particular case the closed-loop system reaches $\Omega$ in four steps, as it can be seen in Figure 1.

Usually, it is not true that the system reaches the target set in $i$ or less steps, for an initial state $x_{0} \in \mathscr{Q}^{i}(\Omega)$. The shape and size of the controllable sets depend on the system and the constraints, while the numbers of steps it takes the controller to steer the system to the target set usually depends on the objectives function of the optimization problem. For instance, if there is a change in the cost function parameters, the controllable sets remains the same but the system reaches the target sets in twelve steps, even when it could do it in four steps, i.e. the initial state $x_{0}=(1,-9 / 2)$ belongs to $\mathscr{Q}^{4}(\Omega)$ (see Figure 2).

\section{C. $i$-Steps closed-loop sets}

The knowledge of the $i$-steps closed-loop sets (Definition 3) is essential in problems in which the time of convergence to a reference zone is important. In fact, these sets indicate how many steps (at most) it would take to the MPC controller to steer the system to the reference zone and Proposition 1 relates

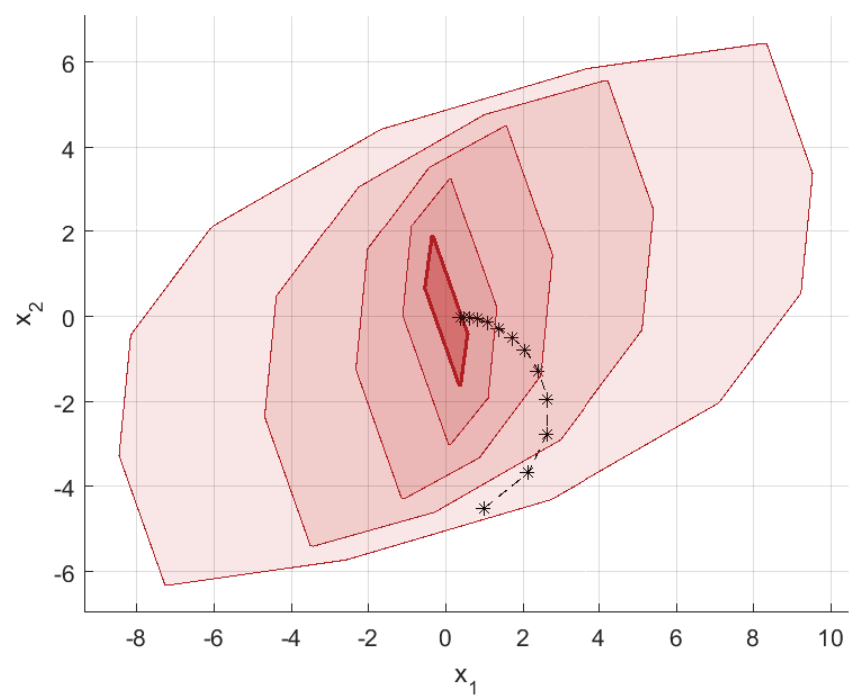

Fig. 2: Controlled system starting in $x_{0}=(1,-9 / 2) \in \mathscr{Q}^{4}(\Omega)$ for which it takes twelve steps to reach the target set $\Omega$.

this maximum number of steps with the optimal cost function, $F(x)$.

A drawback of the method proposed in Proposition 1 is that there is not an explicit form to compute the optimal cost function $F(x)$, given that $F(x)$ implicitly includes an optimization problem with constraints. Furthermore, the dependence on the computation of the upper bound $K_{x}$ for all $x \in \mathscr{X}$, could represents another drawback, given that this upper bound (originally proposed in [1]) could be a bit conservative.

Due to these facts, the $i$-steps closed-loop sets are computed numerically in these simulations. In this regard, note that $F(x)$ is a Lyapunov function and fulfill the following inequalities

$$
\begin{aligned}
& F(x) \leq \alpha d_{\Omega}(x) \\
& F(x) \geq \beta d_{\Omega}(x)
\end{aligned}
$$

where $\alpha, \beta$ are positive scalars. Therefore, $F(x)$ can be approximated by the function $\zeta d_{\Omega}(x)$ for a proper scalar $\zeta$ such that $\beta \leq \zeta \leq \alpha$.

So, in order to observe the properties of the proposed sets, it is still possible to use Proposition 1 by the numerical approach of function $F(x)$, as it is shown in Figure 3 .

Figure 4 shows the same control scenario presented in Figure 1 , but showing the numerically computed $i$-steps closedloop sets $\mathscr{C}^{i}(\Omega)$ instead of the sets $\mathscr{Q}^{i}(\Omega)$. The contour of these sets (plotted in Figure 3) are the level sets of $F(x)$.

Finally, Figure 5 shows the same control scenario of Figure 2; i.e., when the cost function is modified. Note how the $i$-steps closed-loop sets change their shapes according to the new tuning. 

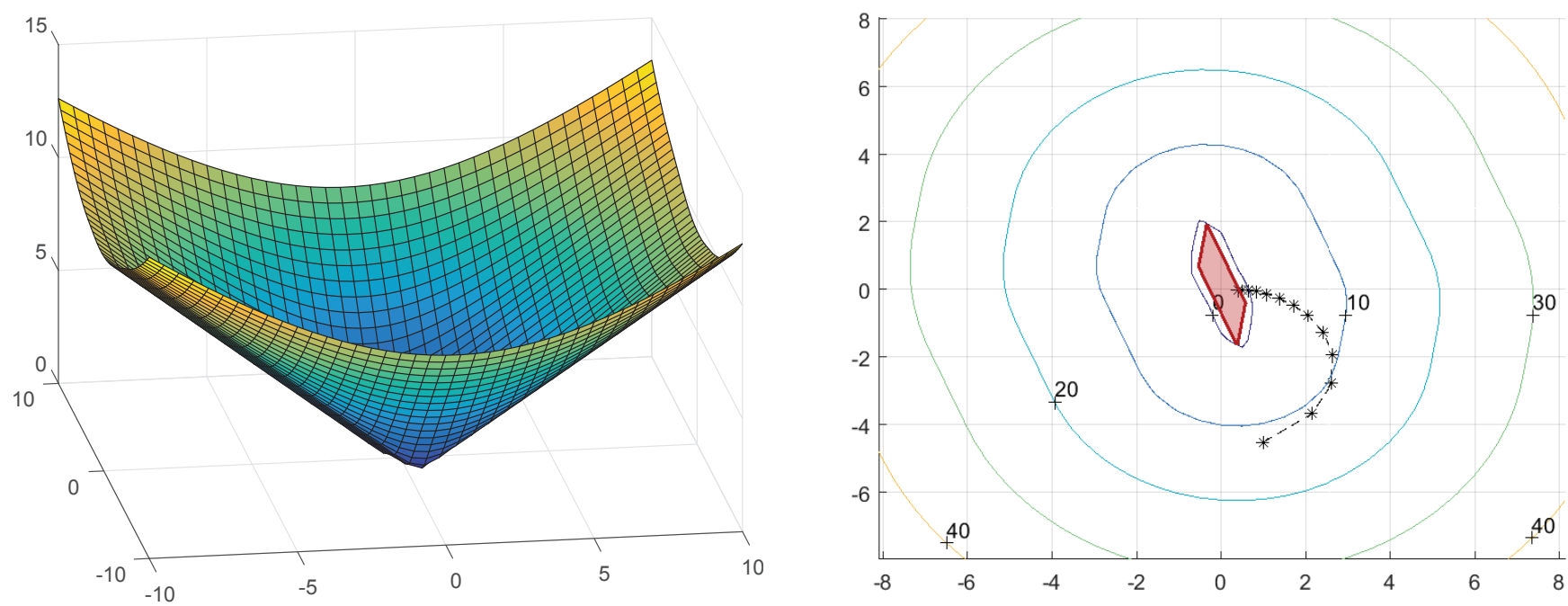

Fig. 3: Numerical approximation of the optimal cost function $F(x)$

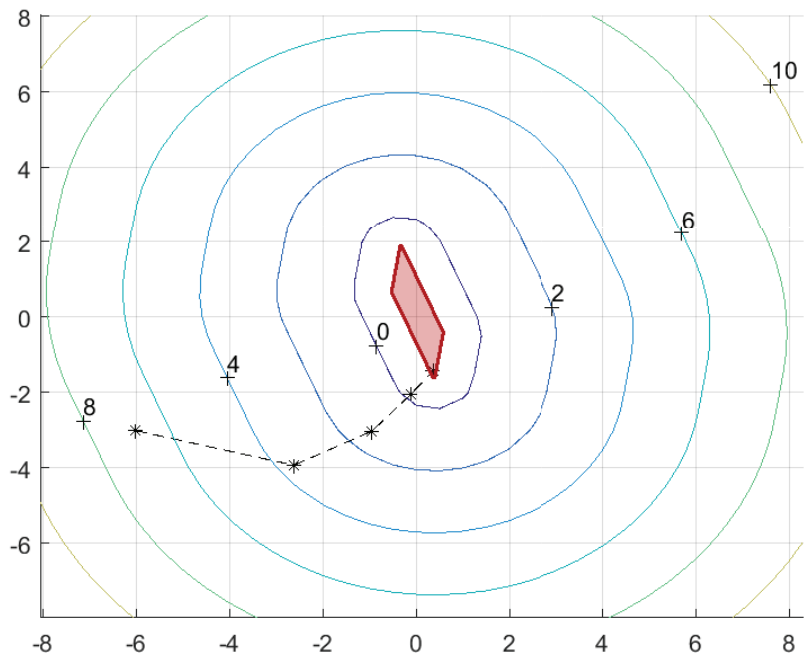

Fig. 4: Controlled system starting in $\mathscr{C}^{8}(\Omega)$ for which it takes four steps to reach the target set $\Omega$.

\section{CONCLUSION}

This work introduced the concept of $i$-steps closed-loop sets, which extends the invariant set and set-based model predictive control theory. These sets are closely related to the well-known $i$-steps controllable sets, which are widely referenced in the classic MPC literature. Additionally, a characterization of these novel sets was made by means of the level sets of the optimal cost function. Some proper simulations show the practical differences - and interesting properties- between the $i$-steps closed-loop set and the $i$-steps controllable sets.
Fig. 5: Controlled system starting in $\mathscr{C}^{20}(\Omega)$ for which it takes twelve steps to reach the target set $\Omega$.

\section{REFERENCES}

[1] A. Anderson, A. H. González, A. Ferramosca, and E. Kofman, "Finitetime convergence results in robust model predictive control," Optimal Control Applications \& Methods, pp. 1627-1637, 2018.

[2] J. B. Rawlings and D. Q. Mayne, Model Predictive Control: Theory and Design, 1st ed. Nob-Hill Publishing, 2009.

[3] D. Q. Mayne, J. B. Rawlings, C. V. Rao, and P. O. M. Scokaert, "Constrained model predictive control: Stability and optimality," Automatica, vol. 36, pp. 789-814, 2000.

[4] F. Blanchini, "Set invariance in control," Automatica, vol. 35, pp. $1747-$ 1767, 1999.

[5] E. C. Kerrigan and J. M. Maciejowski, "Invariant sets for constrained discrete-time systems with application to feasibility in model predictive control," in Proceedings of the CDC, 2000.

[6] P. O. M. Scokaert, D. Q. Mayne, and J. B. Rawlings., "Suboptimal model predictive control (feasibility implies stability)," IEEE Transactions on Automatic Control, vol. 44, no. 3, pp. 648-654, 1999.

[7] E. C. Kerrigan and J. M. Maciejowski, "Feedback min-max model predictive control using a single linear program: robust stability and the explicit solution," International Journal of Robust and Nonlinear Control, vol. 14, no. 4, pp. 395-413, 2004.

[8] F. Blanchini and S. Miani, Set-Theoretic Methods in Control, ser. Systems \& Control: Foundations \& Applications. Springer International Publishing, 2015. [Online]. Available: https://books.google.com.ar/books?id=8a0YCgAAQBAJ

[9] C. E. T. Doréa and J. C. Hennet, "(a,b)-invariant polyhedral sets of linear discrete-time systems," Journal of Optimization Theory and Applications, vol. 103(3), pp. 521-542, 1999.

[10] A. González, A. Ferramosca, G. Bustos, J. Marchetti, M. Fiacchini, and D. Odloak, "Model predictive control suitable for closed-loop reidentification," Systems and Control Letters, vol. 69, pp. 23-33, 2014. 